\title{
Breast Follicular Lymphoma
}

National Cancer Institute

\section{Source}

National Cancer Institute. Breast Follicular Lymphoma. NCI Thesaurus. Code C40377.

A follicular lymphoma that arises from the breast as a primary tumor. 